\title{
Biophilic Urbanism: Making Cities Sustainable through Ecological Design
}

\author{
Edward A. Cook
}

\begin{abstract}
Nature's role in making cities sustainable is undervalued. This paper presents a framework by which natural patterns and processes contribute to making cities sustainable. Biophilic urbanism embraces an awareness of ecosystem services provided through natural process. Awareness is now increasing and there is interest in developing strategies to preserve, restore or reinterpret ecosystems urban contexts. Five types of urban ecosystems are explored to articulate embodied benefits. Preserved ecosystems are remnant natural ecosystems, but may be declining due to proximate urban transformations. Establishing buffers, connectivity and preserving indigenous species are critical design principles and strategies. Restored ecosystems are established through direct intervention to re-create the pre0existing structure and functions. The objective is to replicate appropriate sustainable conditions to ensure connectivity to supporting structure and context. Hybrid ecosystems exist where urban transformation created conditions where preservation and restoration are impractical, but build on remnant structures of ecosystems blending existing attributes with introduced elements acknowledging transformations in context. Synthetic ecosystems result from ecological design establishing viable systems providing ecosystem services, but are not grounded in previously existing ecosystems proximity, structure or function. Regenerated ecosystems have re-established following significant disturbance or transformation and may vary from characteristics of the native ecosystem.
\end{abstract}

Keywords - biophilic urbanism, ecological design, urban ecology, urban design, urban sustainability.

\section{INTRODUCTION}

Increasing concerns about global urbanization and impacts on the environment has lead to the emergence of urban ecology as a topic of increasing interest to ecologists, urban and regional planners/designers, civil engineers, landscape architects, architects, social scientists, and others. Cities impact ecological functions in many ways. Urbanization "fragments, isolates and degrades natural habitat; simplifies homogeneous species composition; disrupts hydrological systems; and modifies energy flow and nutrient cycling." [1] (p. 169) Urban ecosystems and natural ecosystems have similar interactions. Urban ecosystems, however, are a blend of natural and human-created elements. Accordingly, the interactions are significantly affected by human intervention and natural processes. Biophilic urbanism is a useful concept that integrates nature, human biology, planning and design to help create more sustainable urban environments that reduce stress and enhance physical well-being in an effort to create more resilient and sustainable cities $[2,3,4]$.

Edward A. Cook, The Design School, Arizona State University
Integrating natural processes to achieve biophilic urbanism should be an important sustainability goal because: 1) it helps ameliorate human impacts on ecosystems, 2) it enhances ecosystem services, 3 ) it adds biodiversity value to cities, 4) it ensures equitable access to nature and resources, and 5) it maintains a healthy functioning planet for future generations. An important dimension of biophilic urbanism is to allow nature to continue to thrive and manage itself without the infusion of energy and resources to keep urban ecological systems functioning. This can be addressed through the planning and design processes that integrate ecological design concepts. Urban landscapes are a finely structured mosaic of property owners and land uses where competing interests for undeveloped land are intense. Over time, cities have largely been formed as a result of many political, economic, cultural, and physical determinants. Resulting urban forms are an amalgamation of the most resilient human creations and ecological processes. However, nature's deep structure is everpresent in our cities and continues to provide evidence that when ecological processes are ignored in city design, nature's response is to recapture parts of the city either through catastrophic natural events or through incremental change. Often, valuable resources are used in an attempt to hold back the forces of nature or to rebuild urban infrastructure after recurring natural disruptions such as floods, soil movement, or weathering. A main goal of urban ecology is to understand these forces and work together with natural processes to achieve a sustainable future for cities.

The more encompassing concept of biophilic urbanism can be realized through planning strategies such as green networks that can only be implemented on a local. This is where ecological design becomes an essential element of creating a more sustainable future for cities. Nature has provided and does provide us with exceptional examples of functional, efficient ecological systems. We can use these to inform the process of design and restoration of urban ecosystems. The more we know about how nature functions, the better we will be at designing systems that will provide the benefits we seek. We also have to embrace technology along with natural sciences to develop effective strategies for designing urban ecosystems, resulting in a range of ecosystem types from natural to artificial.

Urban ecological design embraces urban ecology as an essential determinant of city form that provides a guiding philosophy for sustainable new urban development and opportunities to retrofit existing urban structure to the ecological patterns that nature has shaped over time. The goal is to preserve or restore the ecological integrity of critical natural systems while allowing for compatible human activities 
and continued productive economic use of the lands. Urban ecological design can be a response to deleterious effects of fragmentation and ecological degradation. Biophilic urbanism employs the development of a coherent system of natural or semi natural landscape elements configured and managed with the objective of maintaining or restoring ecological functions. A principle benefit is that this concept allows nature to thrive and essentially manage itself with minimal infusion of energy and resources to keep urban ecological systems functioning. In some locations, it may be possible to achieve this based largely on the remaining natural ecosystems and habitats; however, in urban settings, ecological restoration will have to be a critical dimensions by designing a variety of hybrid or synthetic ecosystems [5] that mimic natural systems to establish linkages where no natural connections are possible.

\section{II.URBAN ECOSYSTEMS AND ECOSYSTEM SERVICES}

Urban ecosystems provide a range of ecosystem services upon which society relies and can be organized into four categories (UN 2005). The principal reason urban ecological design is important as a planning and design strategy for cities is that there are a variety of ecosystem services $[6,7,8]$ and cultural benefits that can result. Ecosystem services are the result of the function of ecosystems and provide many benefits that we often take for granted. They are derived from nature and are used extensively to maintain our society. We depend on ecosystems services to provide breathable air, clean and plentiful water, food, pharmaceuticals, clothing, fuel, climate, waste disposal, pollination of plants, carbon sequestration and much more. A number of other functions could be identified, but these are some of the most relevant in urban areas. All of these ecosystem services or functions would likely not occur simultaneously. However, there may be several compatible functions with varying levels of priority in certain segments. Ecosystem services can be organized into four categories [9].

1. Supporting services - those necessary for critical production of other ecosystem services including nutrient cycling, dispersal, primary production.

2. Provisioning services - those necessary to produce food, water, minerals, energy and other products for human use.

3. Regulating services - those necessary for carbon sequestration, climate regulation, waste decomposition, air and water purification, pollination and pest/disease control.

4. Cultural services - those necessary for intellectual and spiritual inspiration, cultural development, recreation, scientific discovery, cognitive development and aesthetics.

Urban ecosystems represent an interesting challenge and opportunity for sustainability into cities. We are now just beginning to incorporate urban landscape ecological studies and as the decline of the benefits they have provided to urban populations throughout history become more evident. The challenge is that as a result of fragmentation, invasion of exotic species and general degradation of ecological functions due to human impact, a range of ecosystem services are being lost that urban populations have come to rely upon. Ecologically functional urban ecosystems are critical since society is no longer in a position to move to the next frontier to exploit pristine ecosystems, to obtain fresh water, attain cooling air flows, clean and transform our waste and more.

One of the greatest challenges with urban ecosystems is that significant damage already exists in many cases and to restore the systems to recapture previously existing values is difficult. Urban ecosystems have been and are subjected to a variety of increased levels of environmental stress. They generally exist as highly disturbed systems and may be subject to rapid change in soil and plant cover, temperature and water availability; all essential factors of ecosystem health. Urban ecosystems are also generally characterized by non-native plants and decreased plant density. They are subject to air pollution, road salts and runoff, poor soils, frequent drought, limited sunlight, introduction of toxic substances, loss of habitat and food and frequent disturbance by human activity.

The concept of green networks is a fundamental planning concept of biophilic urbanism that embraces urban ecology as an essential determinant of city form that provides a guiding philosophy for sustainable new urban development and opportunities to retrofit existing urban structure to the ecological patterns that nature has shaped over time. The goal of green networks is to preserve or restore the ecological integrity of critical natural systems while allowing for compatible human activities within the network and continued productive use of adjacent lands. A green network is a system of interconnected or related patches and corridors that provide and sustain ecological values within a human-dominated landscape mosaic. The concept of green networks is a human interpretation of relationships that have occurred in nature since the beginning of time.

Green networks are also a response to deleterious effects of fragmentation and ecological degradation. If a green network is designed as a coherent system of natural or semi natural landscape elements configured and managed with the objective of maintaining or restoring ecological functions, it will provide opportunities for the sustainable use of natural resources and ecosystem services. A principle benefit is that this concept allows nature to thrive and essentially manage itself with minimal infusion of energy and resources to keep urban ecological systems functioning. In some locations, it may be possible to design green networks based largely on the remaining natural ecosystems and habitats; however, in urban settings, ecological restoration will have to be achieved by designing a variety of hybrid or synthetic ecosystems that mimic natural systems to establish linkages where no natural connections are possible.

\section{URBAN ECOSYSTEM TYPOLOGIES}

The potential for urban ecosystems is great, but it is important to take a different view from the past and consider how we can redefine them, rather than attempting to reestablish a pre-existing condition that may be unsustainable, costly and provide fewer ecosystem services. The metric for urban ecosystems should be more heavily weighted with the values of ecosystems services provided, rather than looking for 
naturalness. There are, of course, situations where naturalness and the presence of native species is viable and desirable in an urban context, but in every circumstance, specific consideration needs to be given to the origin, nature and potential of the system in its current condition and context.

Nature has provided and does provide us with exceptional examples of functional, efficient ecological systems. We can use these to inform the process of design and restoration of urban ecosystems. The more we know about how nature functions, the better we will be at designing systems that will provide the benefits we seek. However, we also have to embrace technology along with natural sciences to develop effective strategies for designing urban ecosystems, resulting in a range of ecosystem types from natural to artificial.

Urban ecosystems are generally significantly modified from ecosystems that were formerly present and as such should be considered within a modified set of parameters that include significant human intervention due to the urban context. Urban open space systems contribute to the quality of the urban environment in many ways. The range of open space types includes urban plazas to remnant natural open space or natural parks. Each of these plays an important role in the dynamics of an urban landscape. However, urban open spaces, while valued for their natural qualities are in a precarious position. Research has demonstrated that over time the qualities that are so valued in these "natural" places will deteriorate because of fragmentation from the supporting structure resulting in isolation. Thus, it is important to establish methods for preserving and re-establishing natural qualities in functioning, self-sustaining ecosystems. Following are descriptions of typologies for reconsidering urban ecosystems.

Preserved Ecosystems - Preserved ecosystems in an urban context are often rare, and when present difficult to maintain at a high level of ecological functioning over time. They would typically be in natural or near-natural condition with intact internal landscape structure or content. Supportive connective systems are also important in order to maintain flows and other functions critical to the process of natural regeneration. This is an important dimension of facilitating dynamic development of the ecosystem. Adjacent land uses or landscape character also have significant influence. Land use compatibility or the existence of buffer zones will be important to filter contaminants, limit the intrusion of invasive species and reduce the potential for other deleterious edge effects. The principle aim is to ensure the ecosystem preserves the ability to function and evolve as if it were still existing and functioning as a natural system in a predominantly natural context. While preservation of natural systems in an urban context with no significant impact is impossible, good planning and effective management can do much to facilitate the preservation of something close to the original.

Restored Ecosystems - Restored ecosystems are those that are re-established with similar structure and function as the original system. Typically, the original ecosystem has become degraded, modified, replaced or completely destroyed. The original ecosystem may be used as the model for restoration if there is sufficient knowledge about its former structure and function. If there is insufficient documentation or knowledge about the original system, then another ecosystem with similar conditions as presumed existed in the original can be used as a model. As with a preserved ecosystem, connecting structure that facilitates flows is important to ensure ecological viability. The context should also be evaluated to determine if it is compatible and does not introduce deleterious effects such as increased storm water flow, toxic materials, potential for invasive species and others. In an urban context precise replication of a pre-existing ecosystem is difficult and is uncertain in its infancy. The principle aim should be, to the extent feasible, to allow the restored ecosystem to mimic the original, while accepting that adaptation is required and that over time the ecosystem will naturally evolve and adapt to changing context and functions. Most effective ecosystem restoration projects explore the ecological history to provide sufficient knowledge of the landscape morphology. In this way, it is possible to understand the dynamic nature of the system and appropriately determine what point in the systems evolution should be used as the model for design. The restored ecosystem should be designed so that the structure represents the model, but functions that also previously existed should be restored or preserved so that it acts like the original and is also set in motion so that regenerative capacity is in place.

Hybrid Ecosystems - A hybrid ecosystem exists within a frame or location of a pre-existing ecosystem and restores some of the original ecological functions while accommodating varied landscape context, supporting structure and some functions. In this case, it is recognized that significant changes in context, supporting structure and functions require and alternative approach to preservation or restoration. Because the hybrid ecosystem is within the same frame or location, the inherent characteristics and recurring tendencies of pre-existing natural systems will support the levels of ecological functioning in the ecosystem, working with the deep structure $[10,11]$ to increase the odds of long term sustainability. Generally, the use of ecological design principles (observing and documenting patterns and processes of nature to inform design) is helpful. Other ecosystems that have similar structure and functions may be used as models to provide a design framework. Critical design and management concepts might include the predominant use of native vegetation, re-establishment of flows and connectivity, periodic intervention to manage outcomes, and significant initial introduction of some wildlife.

Synthetic Ecosystems - Synthetic ecosystems establish critical ecosystem functions through ecological design in locations where similar ecosystems did not previously exist. They may be useful to fill a gap to establish ecological functions in a new location and different landscape structure. A synthetic urban ecosystem may also be useful to establish alternative connections within the larger set of urban ecosystems or green network. They exist where there is no opportunity to work with the deep structure in the landscape. They are designed to establish specific ecosystems functions and ecosystem services. They may also provide a variety of 
other social and cultural benefits embracing the concept of multiple-use. Synthetic ecosystems could also be comprised of technological or invented elements that provide certain ecological or "green" values to society. Buildings and other structures, not normally considered as ecological elements, could also be a part of this type of ecosystem. A synthetic ecosystem also contributes to the overall ecological health of a larger system.

Regenerated Ecosystems - Regenerated ecosystems allow for nature to take its course and naturally regenerate an ecosystem that has been previously disturbed, modified or compromised. Depending on the nature of the disturbance, the result of natural regeneration may vary substantially. Sometimes the result is unpredictable. Generally, this is the least costly option ro re-establish ecological values to a disturbed ecosystem. The trade-off with low cost is accepting the unknown outcome. The time required to re-establish something viable is another variable that can vary with the ecosystem. In some desert landscapes natural regeneration can take generations and then the outcome may be of lesser ecological value. In some cases, where there is potential for undesirable invasive species, natural regeneration may not be a desirable strategy for urban ecosystem renewal. Given the range of possibilities, natural regeneration should, however, be considered as a viable option for re-establishing wild areas in cities [12].

\section{CONCLUSION}

The concept of biophilic urbanism begins with the establishment of urban green networks that address the ecological functioning of urban, patches and corridors through ecological design. The best way to ensure the integrity of these systems is to establish the viability of critical ecological systems within the urban landscape context. This requires planning and management at multiple scales. To preserve, rehabilitate or restore the various components of a green network, management strategies must be developed for individual sites on the local level. This will include various types of patches and corridors and the landscape matrix. The viability of any green network or system of interconnected patches and corridors is made possible at the local scale. The types of elements that need to be addressed may include pieces of the main ecological structure of an area (i.e. large remnant patches or corridors) or sites of a more ephemeral nature (i.e. vacant lots). The future value or potential may also need to be addressed with sites in need of regeneration or restoration.

The typologies of urban ecosystems described in this paper can be useful to help understand the inherent characteristics and qualities of urban ecosystems, but can also be a guide for future transformation through ecological design. They are important to be used to gauge the level of intervention or investment that may be appropriate and also to be more explicit about expectations or outcomes. Strategies developed for preservation, restoration, regeneration or with the creation of invented ecosystems, such as hybrid and synthetic, will become more realistic with additional base knowledge and well-articulated planning and design frameworks.

While it is clear that a need exists for maintaining the viability of critical ecological systems in urban areas, it is uncertain whether this can be achieved over the long-term given the current strategies employed in urban planning in many cities. Ecological and urban theories have evolved in different directions and are only now starting to meet at a point in time when many urban areas have deteriorated ecosystem values that require substantial efforts to restore. Numerous perspectives exist on how to conserve existing viable systems and restore those with degraded quality. The concept of biophilic urbanism shows promise and indications are that in proper conditions, cities can become more resilient and sustainable. Linking science, policy, planning, and design is the most promising way to integrate ecology into cities and help provide a foundation for a more sustainable future.

\section{REFERENCES}

[1] Alberti, M. 2005. The effects of urban patterns on ecosystem function. International Regional Science Review, 28:2 pp 168-192.

[2] Browning, W., C. Ryan and J. Clancy. 2014. 14 Patterns of Biophilic Design. New York:Terrapin Bright Green.

[3] Kellert, S., J. Heerwagen and M. Mador. 200. Biophilic Design: The Theory, Science and Practice of Bringing Building to Life. New York: Wiley and Sons.

[4] Beatley, T. 2011. Biophilic Cities: Integrating Nature into Urban Design and Planning. Washington: Island Press https://doi.org/10.5822/978-1-59726-986-5

[5] Cook, E.A. 2013. Urban Ecosystems and the Sustainable Metropolis. In: E.A. Cook and J.J. Lara (Editors). Remaking Metropolis: Global Challenges of the Urban Landscape. London: Routledge. 323 pages.

[6] Daily, G.C. (Editor). 1997. Nature's Services, societal dependence on natural ecosystems. Island press: Washington DC.

[7] Bolund, P. and S. Hunhammar. 1999. Ecosystem services in urban areas. Ecological Economics 29: 293-301. https://doi.org/10.1016/S0921-8009(99)00013-0

[8] Lundy, L. and R. Wade. 2011. Integrating sciences to sustain urban ecosystem services. Progress in Physical Geography 35: 653-669. https://doi.org/10.1177/0309133311422464

[9] United Nations, 2005. Millennium Ecosystem Assessment (MA). Ecosystems and Human Well-Being: Synthesis. Island Press, Washington. 155pp.

[10] Cook, E.A. 2011. Embracing Nature's Deep Structure in Sustainable Urbanism. In: A. Dokart, O. Al Gohari and S. Rab, (Editors). Conservation of Architecture, Urban Areas, Nature \& Landscape: Towards Sustainable Survival of Cultural Landscapes. CSAAR: Amman. Pp 93-104.

[11] Steiner, F.R. 1996. Connecting infrastructure to deep structure. Places. 10:3 pp. 60-61.

[12] Kowarik, I. 2005. Wild Urban Woodlands: Towards a Conceptual Framework. In: Kowarik, I and S. Korner, Editors. Wild Urban Woodlands: New Perspectives in Urban Forestry. Springer: Berlin. https://doi.org/10.1007/b138211 